\title{
GENERALIZED FLOW INVARIANCE FOR DIFFERENTIAL INCLUSIONS
}

\author{
T. GNANA BHASKAR AND V. LAKSHMIKANTHAM
}

Received 6 May 2004; Revised 12 May 2005; Accepted 12 May 2005

We introduce a generalized notion of invariance for differential inclusions, using a proximal aiming condition in terms of proximal normals. A set of sufficient conditions for the weak and strong invariance in the generalized sense are presented.

Copyright (c) 2006 T. G. Bhaskar and V. Lakshmikantham. This is an open access article distributed under the Creative Commons Attribution License, which permits unrestricted use, distribution, and reproduction in any medium, provided the original work is properly cited.

\section{Introduction}

The existence of solutions and flow invariance for differential inclusions are considered in [1] by using a generalized concept of solutions, namely, the Euler solutions of differential equations, without any continuity assumptions. This is done by utilizing a proximal aiming condition in terms of proximal normals. In a recent paper [2], we generalized the concept of proximal normal in the spirit of [3], and then, employing a generalized proximal aiming condition, we proved the existence and flow invariance results for solutions of differential inclusions.

Here in this paper, we consider a generalized notion of invariance, retaining the original notion of proximal normals as in [1], and study the corresponding results for differential inclusions. This generalized notion of flow invariance is useful in studying the solution sets of fuzzy differential equations, which will be considered in a separate paper.

\section{Preliminaries}

Consider the Cauchy problem

$$
x^{\prime}(t)=f(t, x(t)), \quad x\left(t_{0}\right)=x_{0}, \quad t_{0} \geq 0
$$

where $f:\left[t_{0}, T\right] \times \mathbb{R}^{n} \rightarrow \mathbb{R}^{n}$ is any function. 
2 Generalized flow invariance for differential inclusions

Let $\pi=\left\{t_{0}, t_{1}, \ldots, t_{N}=T\right\}$ be a partition of $\left[t_{0}, T\right]$. On the interval $\left[t_{0}, t_{1}\right]$, we consider the differential equation with constant right-hand side

$$
x^{\prime}(t)=f\left(t_{0}, x_{0}\right), \quad x\left(t_{0}\right)=x_{0},
$$

which has a unique solution, $x(t)$ on $\left[t_{0}, t_{1}\right]$. Let $x_{1}=x\left(t_{1}\right)$. Next, consider, on the interval $\left[t_{1}, t_{2}\right]$, the IVP

$$
x^{\prime}(t)=f\left(t_{1}, x_{1}\right), \quad x\left(t_{1}\right)=x_{1} .
$$

We take $x_{2}=x\left(t_{2}\right)=x\left(t_{2}, t_{1}, x_{1}\right)$ as the next node and proceeding in this manner until we get an $\operatorname{arc} x_{\pi}=x_{\pi}(t)$ defined on all of $\left[t_{0}, T\right]$. The notation $x_{\pi}$ is employed to emphasize the role played by the particular partition $\pi$ in defining $x_{\pi}$ which is the Euler Polygonal arc corresponding to the partition $\pi$. The diameter $\mu_{\pi}$ of the partition $\pi$ is given by

$$
\mu_{\pi}:=\max \left\{t_{i}-t_{i-1}: 1 \leq i \leq N\right\} .
$$

By an Euler solution to the IVP (2.1), we mean any arc $x(t)$ which is the uniform limit of the Euler polygonal arcs $x_{\pi_{j}}$, corresponding to some sequence of partitions $\pi_{j}$ such that the diameters $\mu_{\pi_{j}} \rightarrow 0$ as $j \rightarrow \infty$. Clearly, this Euler arc satifies the initial condition $x\left(t_{0}\right)=x_{0}$ and the corresponding number $N_{j}$ of the partition points in $\pi_{j}$ tends to infinity.

The following theorem, concerning the existence of Euler solutions for (2.1), is proved in $[2]$.

Theorem 2.1. Assume that

(1) $f:\left[t_{0}, T\right] \times \mathbb{R}^{n} \rightarrow \mathbb{R}$ and $\|f(t, x)\| \leq g(t,\|x\|),(t, x) \in\left[t_{0}, T\right] \times \mathbb{R}^{n}$, where $g:\left[t_{0}, T\right] \times$ $\mathbb{R}_{+} \rightarrow \mathbb{R}_{+}$is a continuous function, nondecreasing in $(t, u)$

(2) the maximal solution $r(t)=r\left(t, t_{0}, u_{0}\right)$ of the scalar differential equation

$$
u^{\prime}=g(t, u), \quad u\left(t_{0}\right)=u_{0} \geq 0,
$$

exists on $\left[t_{0}, T\right]$.

Then, there exists an Euler solution $x(t)=x\left(t, t_{0}, x_{0}\right)$ of the IVP $(2.1)$ on $\left[t_{0}, T\right]$ which satisfies a Lipschitz condition and any Euler solution of (2.1) has an estimate

$$
\left\|x(t)-x_{0}\right\| \leq r\left(t, t_{0},\left\|x_{0}\right\|\right)-\left\|x_{0}\right\|, \quad t \in\left[t_{0}, T\right] .
$$

Remark 2.2. We can extend the notion of Euler solution of $(2.1)$ on the interval $\left[t_{0}, T\right]$ to $\left[t_{0}, \infty\right)$ provided we define $f$ and $g$ on $\left[t_{0}, \infty\right)$ instead of $\left[t_{0}, T\right]$, assume that the maximal solution on $r(t)$ exists on $\left[t_{0}, \infty\right)$, and show that Euler solution exists on every $\left[t_{0}, T\right]$, $T \in\left(t_{0}, \infty\right)$.

\section{Generalized flow invariance}

Let $S(t), t \in[0, \infty)$ be a family of nonempty closed subsets of $\mathbb{R}^{n}$. Let $x \in \mathbb{R}^{n}$ be such that $(t, x) \notin\{(t, s): s \in S(t)\}$, for all $t \geq 0$. Suppose that, for $t \geq 0$, there exists an $s_{t} \in S(t)$ 
such that

$$
\left\|x-s_{t}\right\|=\left\|(t, x)-\left(t, s_{t}\right)\right\|=\inf \{\|x-\tilde{s}\|: \tilde{s} \in S(t)\}
$$

The set of all such $s_{t} \in S(t)$, for each $t \geq 0$, is denoted by $\operatorname{proj}_{S(t)}(x)$. The vector $\left(t, x-s_{t}\right)$ determines a proximal normal direction to $(t, S(t))$ at $\left(t, s_{t}\right)$. We call any vector $\eta_{t}$ of the form $\left(t, k\left(x-s_{t}\right)\right)$, for any $k \geq 0$, a proximal normal (or P-normal) to $S(t)$ at $s_{t}$, at height $t$. The set of all $\eta_{t}$ obtained in this manner is called a proximal normal cone to $S(t)$ at $s_{t}$, at a height $t$ and is denoted by $N_{S(t)}^{P}\left(s_{t}\right)$. If $s_{t} \in S(t)$ such that $s_{t} \notin \operatorname{proj}_{S(t)}(x)$ for all $(t, x) \notin\{(t, s): s \in S(t)\}$, then we set $N_{S(t)}^{P}\left(s_{t}\right)=\{0\}$. If $s_{t} \notin S(t)$, then $N_{S(t)}^{P}$ is not defined.

Definition 3.1 (generalized flow invariance). The system $\left\{(S(t), f): t \geq t_{0}\right\}$ is said to be weakly invariant if for all $x_{0} \in S\left(t_{0}\right)$, there exists an Euler solution $x(t)$ of $(2.1)$ on $\left[t_{0}, \infty\right)$ such that $x\left(t_{0}\right)=x_{0}$ and $x(t) \in S(t), t>t_{0}$.

Note that this implies $(t, x(t)) \in(t, S(t)), t \geq t_{0}$. Also, if $S(t)=S\left(t_{0}\right)$, for all $t \geq t_{0}$, then the above notion of weak invariance coincides with the one given in [1].

Throughout the rest of the paper, we make the following assumption.

Assumption 3.2. For all $t>\tau, t, \tau \in\left[t_{0}, \infty\right)$ and $z \in \mathbb{R}^{n}$,

$$
d_{S(t)}^{2}(z) \leq d_{S(\tau)}^{2}(z)+(t-\tau)^{2}
$$

We can now prove the following result which provides sufficient conditions in terms of the generalized proximal normal for weak invariance of $\{(S(t), f): t \geq 0\}$.

Theorem 3.3. Let $f$ and $g$ satisfy the assumptions of Theorem 2.1 on $\left[t_{0}, \infty\right)$ and let $x(t)$ be an Euler solution on $\left[t_{0}, \infty\right)$ of (2.1). Suppose that $x(t)$ lies in an open set $\Omega \subset \mathbb{R}^{n}$. Assume that for every $(t, z) \in\left[t_{0}, \infty\right) \times \omega$, there exists an $s_{t} \in \operatorname{proj}_{S(t)}(z)$ such that

$$
2\left\langle f(t, z),\left(z-s_{t}\right)\right\rangle \leq q\left(t, d_{S(t)}^{2}(z)\right)
$$

where $q \in C\left(\left[t_{0}, \infty\right) \times \mathbb{R}_{+}, \mathbb{R}\right)$. Suppose also that the maximal solution $r(t)=r\left(t, t_{0}, u_{0}\right)$ of the scalar differential equation $u^{\prime}=q(t, u), u\left(t_{0}\right)=u_{0} \geq 0$ exists on $\left[t_{0}, \infty\right)$. Then,

$$
d_{S(t)}(x(t)) \leq r\left(t, t_{0}, d_{S\left(t_{0}\right)}^{2}\left(x_{0}\right)\right)
$$

If, in addition, $r\left(t, t_{0}, 0\right) \equiv 0$, then $(S(t), f), t \geq t_{0}$, is weakly invariant.

Proof. Let $x_{\pi}(t)$ be one polygonal arc in the sequence, converging uniformly to $x$ as per the definiton of Euler solution of (2.1). We denote, as before, its nodes at $t_{i}$ by $x_{i}, i=$ $0,1, \ldots, N$, and hence $x\left(t_{0}\right)=x_{0}$. Let $x_{\pi}(t)$ be in $\Omega$ for all $t_{0} \leq t \leq T$, where $T \in\left(t_{0}, \infty\right)$. Accordingly, there exists for each $i$ an element $s_{t_{i}} \in \operatorname{proj}_{s\left(t_{i}\right)}\left(x_{i}\right)$ such that

$$
2\left\langle f\left(t_{i}, x_{i}\right), x_{i}-s_{t_{i}}\right\rangle \leq q\left(t_{i},\left\|x_{i}-s_{t_{i}}\right\|^{2}\right) .
$$


4 Generalized flow invariance for differential inclusions

As in Theorem 2.1, letting $\left\|x_{\pi}^{\prime}\right\| \leq k$, we find

$$
\begin{aligned}
d_{S\left(t_{1}\right)}^{2}\left(x_{1}\right) & \leq d_{S\left(t_{0}\right)}^{2}\left(x_{1}\right)+\left(t_{1}-t_{0}\right)^{2} \\
& \leq\left\|x_{1}-s_{t_{0}}\right\|^{2}+\left(t_{1}-t_{0}\right)^{2} \\
& \leq\left(t_{1}-t_{0}\right)^{2}+\left\|x_{1}-x_{0}\right\|^{2}+\left\|x_{0}-s_{t_{0}}\right\|^{2}+2\left\langle x_{1}-x_{0}, x_{0}-s_{t_{0}}\right\rangle \\
& \leq\left(k^{2}+1\right)\left(t_{1}-t_{0}\right)^{2}+d_{S\left(t_{0}\right)}^{2}\left(x_{0}\right)+2 \int_{t_{0}}^{t_{1}}\left\langle x_{\pi}^{\prime}(t), x_{0}-s_{t_{0}}\right\rangle d t \\
& =\left(k^{2}+1\right)\left(t_{1}-t_{0}\right)^{2}+d_{S\left(t_{0}\right)}^{2}\left(x_{0}\right)+2 \int_{t_{0}}^{t_{1}}\left\langle f\left(t_{0}, x_{0}\right), x_{0}-s_{t_{0}}\right\rangle d t \\
& \leq\left(k^{2}+1\right)\left(t_{1}-t_{0}\right)^{2}+d_{S\left(t_{0}\right)}^{2}\left(x_{0}\right)+q\left(t_{0}, d_{S\left(t_{0}\right)}^{2}\left(x_{0}\right)\right)\left(t_{1}-t_{0}\right) .
\end{aligned}
$$

Since similar estimates hold at any node, we have for $i=1,2, \ldots, N$,

$$
d_{S\left(t_{i}\right)}^{2}\left(x_{i}\right) \leq d_{S\left(t_{i-1}\right)}^{2}\left(x_{i-1}\right)+\left(k^{2}+1\right)\left(t_{i}-t_{i-1}\right)^{2}+q\left(t_{i-1}, d_{S\left(t_{i-1}\right)}^{2}\left(x_{i-1}\right)\right)\left(t_{i}-t_{i-1}\right) .
$$

And therefore, it follows that

$$
\begin{aligned}
d_{S\left(t_{i}\right)}^{2}\left(x_{i}\right) & \leq d_{S\left(t_{0}\right)}^{2}\left(x_{0}\right)+\left(k^{2}+1\right) \sum_{j=1}^{i}\left(t_{j}-t_{j-1}\right)^{2}+\sum_{j=1}^{i} q\left(t_{j-1}, d_{S\left(t_{j-1}\right)}^{2}\left(x_{j-1}\right)\right)\left(t_{j}-t_{j-1}\right) \\
& \leq d_{S\left(t_{0}\right)}^{2}\left(x_{0}\right)+\left(k^{2}+1\right) \mu_{\pi} \sum_{j=1}^{i}\left(t_{j}-t_{j-1}\right)^{2}+\sum_{j=1}^{i} q\left(t_{j-1}, d_{S\left(t_{j-1}\right)}^{2}\left(x_{j-1}\right)\right)\left(t_{j}-t_{j-1}\right) \\
& \leq d_{S\left(t_{0}\right)}^{2}\left(x_{0}\right)+\left(k^{2}+1\right)\left(T-t_{0}\right) \mu_{\pi}+\sum_{j=1}^{i} q\left(t_{j-1}, d_{S\left(t_{j-1}\right)}^{2}\left(x_{j-1}\right)\right)\left(t_{j}-t_{j-1}\right) .
\end{aligned}
$$

We now consider the sequence $x_{\pi_{j}}(t)$ of polygonal arcs converging to $x(t)$. Since the last estimate is true at every node, $\mu_{\pi_{j}} \rightarrow 0$ as $j \rightarrow \infty$, and the same $k$ applies to each $x_{\pi}$, we deduce in the limit the integral inequality

$$
d_{S(t)}^{2}(x(t)) \leq d_{S\left(t_{0}\right)}^{2}\left(x_{0}\right)+\int_{t_{0}}^{t} q\left(\tau, d_{S(\tau)}^{2}(x(\tau))\right) d \tau, \quad t_{0} \leq t \leq T,
$$

which is the same as

$$
d_{S(t)}^{2}(x(t)) \leq r\left(t, t_{0}, d_{S\left(t_{0}\right)}^{2}\left(x_{0}\right)\right) .
$$

If $r\left(t, t_{0}, 0\right) \equiv 0$, then assuming $x_{0} \in S\left(t_{0}\right)$ implies $x(t) \in S(t)$ for $t \geq t_{0}$ and therefore the system $(S(t), f), t \geq t_{0}$, is weakly invariant. The proof is complete.

\section{Weak invariance for differential inclusions}

Consider the IVP for the differential inclusion

$$
x^{\prime} \in F(t, x), \quad x\left(t_{0}\right)=x_{0},
$$


where $F$ satisfies the following hypotheses:

(a) $F$ is a nonempty convex set for each $(t, x) \in \mathbb{R}_{+} \times \mathbb{R}^{n}$;

(b) $F$ is upper semicontinuous;

(c) $v \in F(t, x)$ implies that $\|v\| \leq g(t,\|x\|)$, where $g \in C\left[\mathbb{R}_{+}^{2}, \mathbb{R}_{+}\right], g(t, w)$ is nondecreasing in $w$, and the maximal solution $r(t)=r\left(t, t_{0}, w_{0}\right)$, of the scalar differential equation

$$
w^{\prime}=g(t, w), \quad w(0)=w_{0} \geq 0,
$$

exists on $[0, \infty)$.

We recall the notions of lower and upper Hamiltonians, which are functions from $\mathbb{R} \times$ $\mathbb{R}^{n} \times \mathbb{R}^{n}$ as follows:

$$
h(t, x, p)=\min _{v \in F(t, x)}\langle p, v\rangle, \quad H(t, x, p)=\max _{v \in F(t, x)}\langle p, v\rangle .
$$

We are now in a position to discuss the existence and weak invariance of $(\mathrm{S}(t), \mathrm{F})$.

Theorem 4.1. Assume that for each $t \geq t_{0}$ and every $s_{t} \in S(t)$,

$$
h\left(t, s_{t}, N_{S(t)}^{P}\left(s_{t}\right)\right) \leq 0 .
$$

Suppose further that $g(t, u)$ is subadditive in $u$, for each $t$. Then the system $(S(t), F), t \geq t_{0}$, is weakly invariant.

Proof. For each $t \in\left[t_{0}, \infty\right)$ and $x \in \mathbb{R}^{n}$, choose $s_{t}=s_{t}(x) \in \operatorname{proj}_{S(t)}(x)$, and let $v_{t}$ in $F\left(t, s_{t}\right)$, minimize over $F\left(t, s_{t}\right)$ the function $v_{t} \rightarrow\left\langle v_{t}, x-s_{t}\right\rangle$.

Set $f_{p}(t, x)=v_{t}$. Since $x-s_{t} \in N_{S(t)}^{P}\left(s_{t}\right)$, we have $\left\langle f_{P}(t, x), x-s_{t}\right\rangle \leq 0$. This implies that the main assumption of Theorem 3.3 with $q(t, u)=0$ is satisfied. If $s_{0} \in S\left(t_{0}\right)$ is a given element, then for each $t \geq t_{0}$,

$$
\begin{aligned}
\left\|f_{p}(t, x)\right\|=\left\|v_{t}\right\| & \leq g\left(t,\left\|s_{t}\right\|\right)=g\left(t,\left\|s_{t}-x+x\right\|\right) \\
& \leq g\left(t,\left\|s_{t}-x\right\|\right)+g(t,\|x\|) \\
& \leq g\left(t,\left\|s_{0}-x\right\|\right)+g\left(t,\left|t-t_{0}\right|^{2}\right)+g(t,\|x\|) \\
& \leq 2 g(t,\|x\|)+g\left(t,\left\|s_{0}\right\|\right)+g\left(t,\left|t-t_{0}\right|^{2}\right)=\tilde{g}(t,\|x\|) .
\end{aligned}
$$

Clearly $\tilde{g}(t, u) \in C\left(\left[t_{0}, T\right] \times \mathbb{R}_{+}, \mathbb{R}_{+}\right)$and $\tilde{g}(t, u)$ is nondecreasing in $(t, u)$. Thus $f_{p}(t, x)$ satisfies the nonlinear growth condition required by Theorem 2.1. Thus, by Theorem 3.3, for any $x(0)=x_{0}$, we have $x(t) \in S(t)$, on $\left[t_{0}, \infty\right)$.

The proof will be complete if we show that $x(t)$ is a solution of (4.2). Since $f_{P}$ is not a selection of $F$, let us define another multifunction as follows:

$$
\text { for each } t \geq t_{0}, \quad F_{S(t)}(t, x)=\operatorname{co}\left\{F\left(t, s_{t}\right): s_{t} \in \operatorname{proj}_{S(t)}(x)\right\} .
$$

It can be verified that $f_{P}(t, x)$ is a selection for $F_{S(t)}(t, x)$, that $F_{S(t)}$ satisfies the hypothesis made at the beginning of this section, and that $F_{S(t)}(t, x)=F(t, x)$ for $x \in S(t)$. Since we know that an Euler solution $x(t)$ of any selection $f_{p}$ of $F_{S(t)}$ is also a solution of (4.2), it follows that $x^{\prime}(t) \in F_{S(t)}(t, x(t))$ a.e. Since $F=F_{S(t)}$ on $S(t)$ and $x(t) \in S(t), t \geq t_{0}$, it 
6 Generalized flow invariance for differential inclusions

follows that $x(t)$ is a solution of (4.2), and therefore $(S(t), F)$ is weakly invariant. The proof is complete.

\section{Strong invariance}

We begin with the following definiton.

Definition 5.1. The multifunction $F$ is said to be locally Lipschitz in $x$, uniformly in $t$, provided that for all $t \in\left[t_{0}, \infty\right)$, each $x \in \mathbb{R}^{n}$ admits a neighborhood $U=U(x)$ and a positive constant $K=K(x)$ such that

$$
x_{1}, x_{2} \in U \Longrightarrow F\left(t, x_{2}\right) \subseteq F\left(t, x_{1}\right)+K\left\|x_{1}-x_{2}\right\| \bar{B}
$$

where $\bar{B}$ is the closed unit ball, centred at 0 .

For the remainder of this section, we make the following assumption, which is stronger than Assumption 3.2.

Assumption 5.2. For all $t>\tau, t, \tau \in\left[t_{0}, \infty\right)$, and $z \in \mathbb{R}^{n}$,

$$
d_{S(t)}(z) \leq d_{S(\tau)}(z)
$$

Theorem 5.3. Let $(S(t), F)$ be weakly invariant and let $F$ be locally Lipschitz in $x$. Then there exists a feedback selection $g_{P}$ for $F$ under which $S(t)$ is invariant.

Proof. Let $f_{P}(t, x)$ be defined as in Theorem 4.1. Then, $f_{p}(t, x)$ lies in $F\left(s_{t}\right)$, where $s_{t} \in$ $\operatorname{proj}_{S(t)}(x)$. Define, for each $t \geq t_{0}, g_{P}(t, x)$ to be an element in $F(T, x)$ closest to $f_{P}(T, x)$ so that $g_{P}$ is a selection for $F$.

Now, suppose $x_{0} \in S\left(t_{0}\right)$ and $\left[t_{0}, T\right]$ is any interval. We will show that any Euler solution $y(t)$ on $\left[t_{0}, T\right]$ from $x_{0}$ generated by $g_{P}$ is such that $y(t) \in S(t), t \in\left[t_{0}, T\right]$. We know there is a bound for $y(t)$ on $\left[t_{0}, T\right]$ such that $\left\|y(t)-x_{0}\right\|<M$. Let $K$ be the Lipschitz constant for $F$ on $B\left[x_{0}, M_{0}\right]$.

If $\left\|x-x_{0}\right\|<M$, then

$$
\begin{aligned}
\left\|s_{t}-x_{0}\right\| & \leq\left\|s_{t}-x\right\|+\left\|x-x_{0}\right\| \\
& =d_{S(t)}(x)+\left\|x-x_{0}\right\| \\
& \leq d_{S\left(t_{0}\right)}\left(x_{0}\right)+\left|t-t_{0}\right|+\left\|x-x_{0}\right\| \\
& \leq 2|| x-x_{0} \|+\left|T-t_{0}\right| \\
& <M_{0} .
\end{aligned}
$$

Since $\left\langle s_{p}(t, x), x-s_{t}\right\rangle \leq 0$, we obtain the following estimate:

$$
\begin{aligned}
\left\langle g_{P}(t, x), x-s_{t}\right\rangle & =\left\langle f_{P}(t, x), x-s_{t}\right\rangle+\left\langle g_{P}(t, x)-f_{P}(t, x), x-s_{t}\right\rangle \\
& \leq\left\|g_{P}(t, x)-f_{P}(t, x)\right\|\left\|\mid x-s_{t}\right\|^{2} \\
& =K d_{S(t)}^{2}(x) .
\end{aligned}
$$


Thus, by [1, Exercise 2.2], and an application of Gronwall inequality, we get

$$
d_{S(t)}(y(t)) \leq d_{S\left(t_{0}\right)}\left(x_{0}\right) e^{K t}, \quad t \in\left[t_{0}, T\right] .
$$

Since $x_{0} \in S\left(t_{0}\right)$, this implies that $y(t) \in S(t), t \in\left[t_{0}, T\right], T \in\left(t_{0}, \infty\right)$.

We can now prove the strong invariance of the system $(S(t), F)$.

Theorem 5.4. Let $F$ be locally Lipschitz and suppose that for each $t \geq t_{0}$ and every $s_{t} \in S(t)$,

$$
H\left(t, x, N_{S(t)}\left(s_{t}\right)\right) \leq 0, \quad \forall S(t) .
$$

Then, $(S(t), F), t \geq t_{0}$, is strongly invariant.

Proof. Let $y(t)$ be any solution for $F$ on $\left[t_{0}, T\right]$ for each $t$, with $y\left(t_{0}\right)=x_{0} \in S(0)$. As a consequence of Theorem 5.3, there exists an $f$ such that $y(t)$ is an Euler solution of the IVP $x^{\prime}=f(t, x), x\left(t_{0}\right)=x_{0}$. As in Theorem 5.3, if $M>0$ is such that all Euler solutions $x(t)$ of this IVP satisfy $\left\|x(t)-x_{0}\right\|<M$, then $\left\|s_{t}-x\right\| \leq M_{0}$, where $s_{t} \in \operatorname{proj}_{S(t)}(x)$. This means that $s_{t} \in B\left(x_{0}, M_{0}\right)$.

Let $K$ be the Lipschitz constant for $F$ on $B\left(x_{0}, M_{0}\right)$ and consider any $x \in B\left(x_{0}, M_{0}\right)$ and $s_{t} \in \operatorname{proj}_{S(t)}(x)$. Then, $x-s_{t} \in N_{S(t)}^{P}\left(s_{t}\right)$. Since $f(t, x) \in F(t, x)$, there exists $v \in F\left(t, s_{t}\right)$ so that

$$
\|v-f(t, x)\| \leq K\left\|s_{t}-x\right\|=K d_{S(t)}(x)
$$

This leads us to

$$
\left\langle f(t, x), x-s_{t}\right\rangle \leq K d_{S(t)}^{2}(x)
$$

Using an argument similar to Theorem 5.3, we conclude that $y(t) \in S(t), t \in\left[t_{0}, T\right]$, since $x_{0} \in S\left(t_{0}\right)$. Since $T \in\left(t_{0}, \infty\right)$, we have that $(S(t), F), t \geq t_{0}$, is strongly invariant and the proof is complete.

\section{References}

[1] F. H. Clarke, Yu. S. Ledyaev, R. J. Stern, and P. R. Wolenski, Nonsmooth Analysis and Control Theory, Graduate Texts in Mathematics, vol. 178, Springer, New York, 1998.

[2] T. Gnana Bhaskar and V. Lakshmikantham, Generalized proximal normals and flow invariance for differential inclusions, Nonlinear Studies 10 (2003), no. 1, 29-37.

[3] G. S. Ladde and V. Lakshmikantham, On flow-invariant sets, Pacific Journal of Mathematics 51 (1974), 215-220.

T. Gnana Bhaskar: Department of Mathematical Sciences, Florida Institute of Technology,

Melbourne, FL 32901, USA

E-mail address: gtenali@fit.edu

V. Lakshmikantham: Department of Mathematical Sciences, Florida Institute of Technology,

Melbourne, FL 32901, USA

E-mail address: lakshmik@fit.edu 


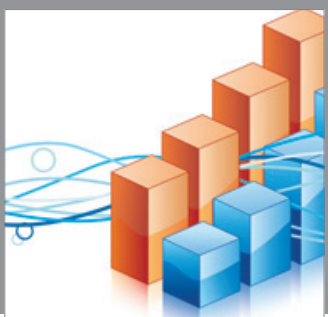

Advances in

Operations Research

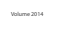

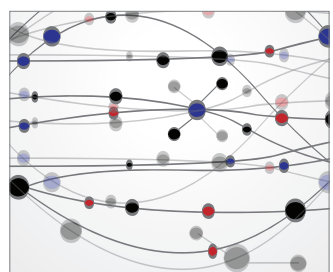

\section{The Scientific} World Journal
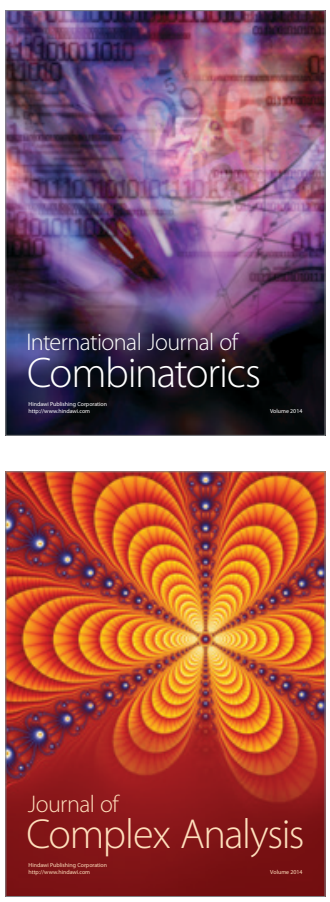

International Journal of

Mathematics and

Mathematical

Sciences
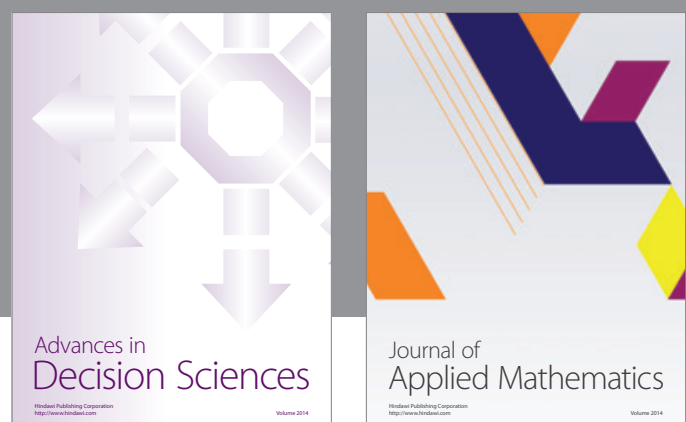

Journal of

Applied Mathematics
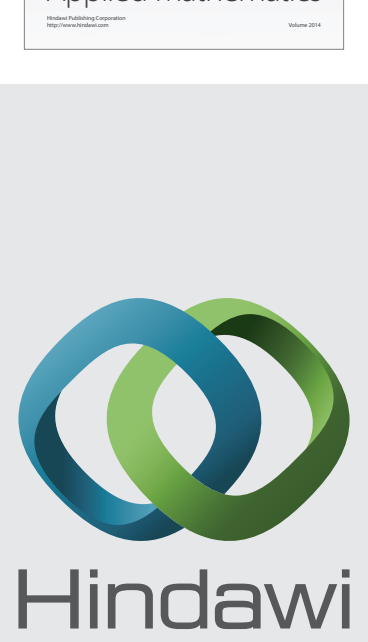

Submit your manuscripts at http://www.hindawi.com
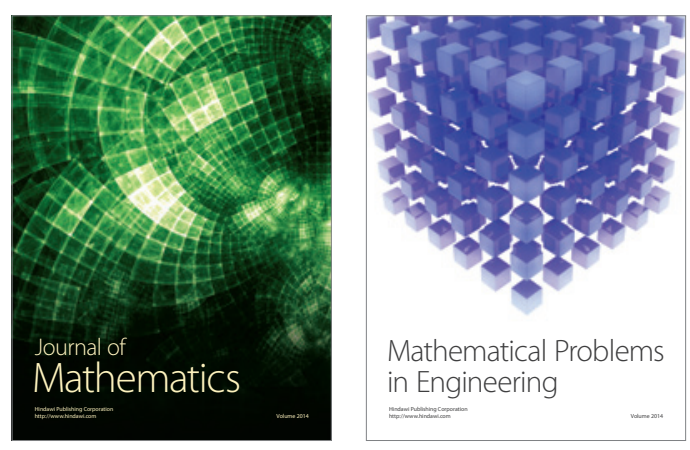

Mathematical Problems in Engineering
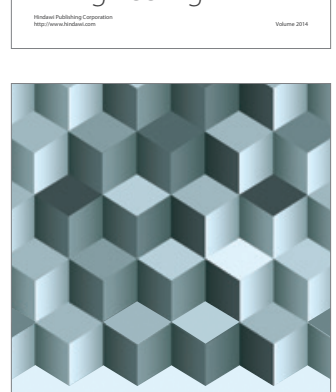

Journal of

Function Spaces
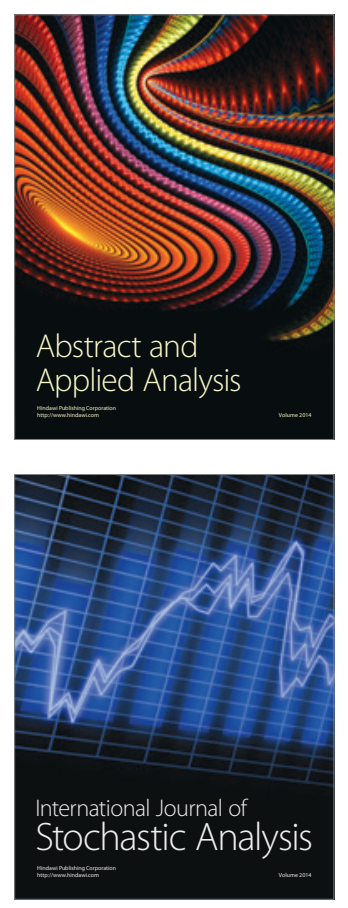

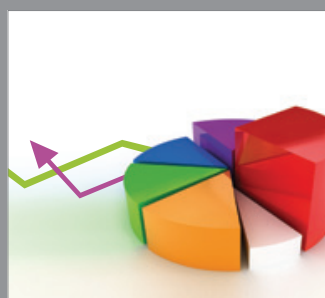

ournal of

Probability and Statistics

Promensencen
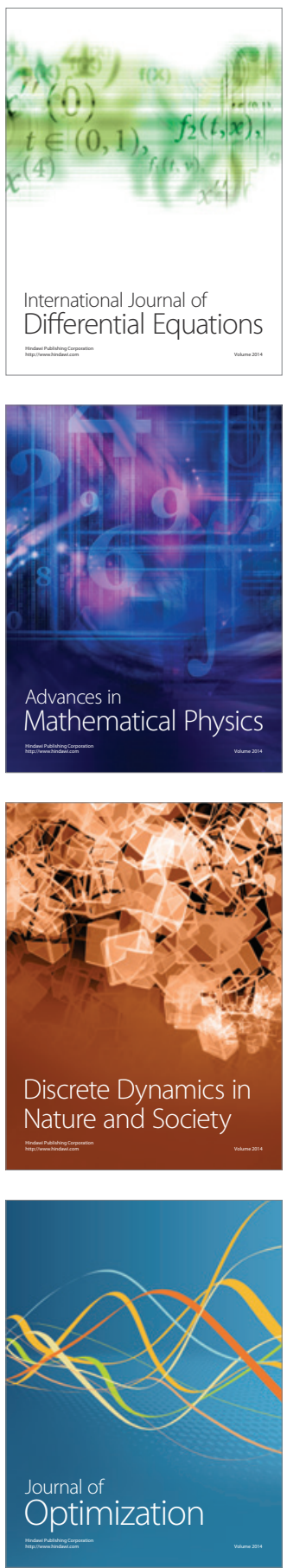